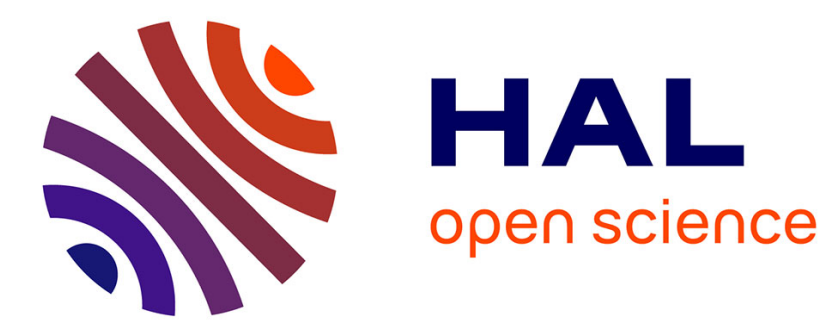

\title{
Model of Crack Propagation in Heterogeneous Materials Local Approach
}

\author{
P. Daguier, E. Bouchaud, G. Lapasset
}

\section{To cite this version:}

P. Daguier, E. Bouchaud, G. Lapasset. Model of Crack Propagation in Heterogeneous Materials Local Approach. Journal de Physique IV Proceedings, 1996, 06 (C6), pp.C6-483-C6-490. 10.1051/jp4:1996648 . jpa-00254481

\section{HAL Id: jpa-00254481 https://hal.science/jpa-00254481}

Submitted on 1 Jan 1996

HAL is a multi-disciplinary open access archive for the deposit and dissemination of scientific research documents, whether they are published or not. The documents may come from teaching and research institutions in France or abroad, or from public or private research centers.
L'archive ouverte pluridisciplinaire HAL, est destinée au dépôt et à la diffusion de documents scientifiques de niveau recherche, publiés ou non, émanant des établissements d'enseignement et de recherche français ou étrangers, des laboratoires publics ou privés. 


\title{
Model of Crack Propagation in Heterogeneous Materials Local Approach
}

\author{
P. Daguier, E. Bouchaud and G. Lapasset \\ ONERA (OM), 29 avenue de la Division Leclerc, BP. 72, 92322 Châtillon cedex, France
}

\begin{abstract}
A continuous numerical model of pure mode I crack propagation in a bidimensional heterogeneous material is presented. This model describes the propagation of a macrocrack into a two-phases brittle material with a finite density of inclusions. The morphology of cracks produced for several mechanical and microstructural conditions is analysed. We find that the simulated cracks are self-affine. Furthermore, it is shown that there exists a microstructure leading to an optimal fracture toughness.
\end{abstract}

\section{INTRODUCTION}

It is now established that fracture surfaces can be considered as self-affine fractal objects $[1,2,3]$. Although many experiments made on metallic alloys $[4,5]$, rocks $[6,7]$, glass [8] or numerical simulations [9] report a roughness exponent $\zeta$ (related to the fractal dimension of the surface) which seems independant of the material $(\zeta \simeq 0.8)$, the universality of this index is still controversial $[10,11]$.

As the roughness exponent seems to be independant of the material, the link between fracture toughness and microstructure should be found elsewhere. By rewritting the Griffith criterion for a self-affine crack, Bouchaud et al. [12] argued that the fracture toughness is related to the spikiness $h m a x / \xi$ of the fracture surface, where $\xi$ is the self-affine correlation length (i.e. the limit above which a fracture surface is flat) and hmax is the typical heigth on the fracture surface outside the fractal regime.

The central point of this paper is to deal with the influence of the microstructural parameters on the morphology of the simulated cracks and hence on the fracture toughness. So, a continuous numerical model of pure mode I crack propagation in a bidimensional heterogeneous material is built up. The principles of the simulation are presented in the next section. The results of the simulations will be reported in section 3 , and discussed in section 4 .

On the other hand, Millman et al. $[13,14]$ reported significantly smaller exponents $(\zeta \simeq 0.4-0.6)$ for fracture surfaces of metallic materials observed with a scanning tuneling microscope (STM) in the nanometer range. For low cycle fatigue, this regime has been observed in the micrometer range by Mc Anulty et al. [15]. Recently, it has been proposed that the lower exponent is characteristic of a new regime that could be identified to "quasi-static" fracture [5, 16]. This interpretation is strengthened by theoretical predictions concerning the morphology of a moving line through randomly distributed impurities [17, 18, 19]. Moreover, recent molecular dynamics simulations on the fracture of amorphous materials report a "large length scales / high velocity" regime with a characteristic exponent close to 0.8 and a "small length scales / small velocity" regime with $\zeta \simeq 0.44$ [20].

The model proposed here indicates the existence of a similar scenario, although our samples are still too small to allow for a proper determination of the two regimes. 


\section{COMPUTATIONAL PROCEDURE}

\subsection{Description of the model}

We consider the propagation of a macrocrack lying along the $x$-axis under a purely Mode I loading condition. The crack, after running over a distance $l o=5000$, enters into a box of dimensions (xl,yl). This box contains a finite number of sites $N$ (which positions are randomly distributed with a uniform probability law) where microcracks may nucleate. A cleavage type criterion is chosen for the nucleation of microcracks: if the stress produced by the macrocrack at site $i$ overcomes a critical stress $\sigma_{C}(i)$, then a microcrack of length so nucleates. The threshold cleavage stresses $\sigma_{C}(i)$ are random variables, distributed with a uniform probability law. The stress acting on site $i$ is given by:

$$
\sigma_{i}=\frac{k_{I}}{\sqrt{\pi d_{i}}}
$$

where $d_{i}$ is the distance between the macrocrack tip and site $i . k_{I}$ is the opening stress intensity factor (SIF) at the tip of the macrocrack in the direction of crack propagation. The computation of $k_{I}$ takes into account both the macrocrack deflection and the presence of the microcracks.

Once opened, the cleavage microcracks may progress towards the main crack tip if the local SIF overpasses a threshold $K_{I}^{\text {th }}$ for propagation into the matrix. In order to take into account the presence of heterogeneities in a "mean field" type of approximation, the microcrack follows a directed random walk to the main crack tip.

If the distance between the tips of the macrocrack and microcrack $i$ is smaller than a distance $R_{\text {plasto }}$, they coalesce instantaneously. The back tip of the microcrack then becomes the new main crack tip. After the microcraks' nucleation / growth stage, the modified SIFs of the main crack tip, due to the presence of microcracks, are computed assuming that the main crack is approximately straigth and under mode I and that there is no interaction between the microcracks.

The computation of the new SIFs at the main crack tip is based on fist order analytical results due to Rose [21]. The modification of the SIFs due to the presence of microcracks is mainly governed by (see Fig 1):

- the ratio between the length of microcrack number $i, 2 s_{i}$, and its distance to the main crak tip $r_{i}$

- the $i^{t h}$ microcrack position and orientation defined by two angles $\theta_{i}$ and $\alpha_{i}$.

$$
\left\{\begin{array}{l}
K_{I}=K_{I, 0}\left[1+\sum_{i=1}^{N_{\text {open }}} \mathcal{F}\left(\frac{s_{i}}{r_{i}}, \theta_{i}, \alpha_{i}\right)\right] \\
K_{I I}=K_{I, 0} \quad \sum_{i=1}^{N_{\text {open }}} \mathcal{G}\left(\frac{s_{i}}{r_{i}}, \theta_{i}, \alpha_{i}\right)
\end{array}\right.
$$

where $K_{I, 0}$ is the remote $\mathrm{SIF}, \mathcal{F}$ and $\mathcal{G}$ are first order pertubative terms, and $N_{\text {open }}$ is the number of microcracks.

The creation of a local non-zero $K_{I I}$ produces crack deflection. The crack will propagate in the direction where $k_{I I}(\theta)=0$ or $\frac{\partial k_{I}(\theta)}{\partial \theta}=0$, where $\theta$ is the kink angle. Within the Cotterell-Rice approximation [22], this two criteria give the same result:

$$
\frac{K_{I I}}{K_{I}}=-\operatorname{tg}(\theta / 2) \frac{\cos ^{2}(\theta / 2)}{3 \cos ^{2}(\theta / 2)-2}
$$

For $\theta$ less than 30 degrees, (3) can be reduced to $\frac{K_{L I}}{K_{I}}=-\operatorname{tg}(\theta / 2)$ 


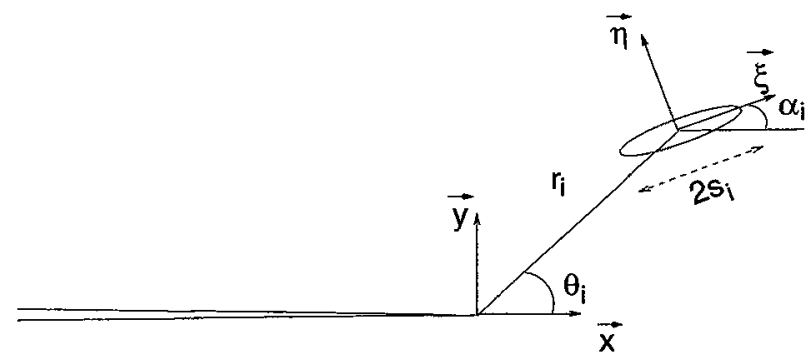

Figure 1: Interaction between the macrocrack and the $i^{\text {th }}$ microcrack

Within the Cotterell-Rice approximation [22], the kink has a length tending to zero. When the ratio of the length of the kink to the length of the crack increases, Kitagawa et al. [23] showed that the opening SIF decreases. This correction is taken into account in the simulation.

As the main crack propagates into the box, more microcracks are produced or grown. The damage produced generates a decrease of the elastic moduli $\nu$ (Poisson modulus) and $\mu$ (shear modulus) of the material following (for $f \ll 1$ ):

$$
\left\{\begin{array}{l}
\frac{\bar{\nu}}{\nu} \simeq 1-\pi f \\
\frac{\mu}{\mu} \simeq 1+\frac{\pi f}{1-\nu}
\end{array}\right.
$$

where $f=\sum_{i=l}^{N_{o p e n}} \frac{s_{i}^{2}}{x_{l} y_{l}}$ is a microcrack density parameter and $\bar{\nu}, \bar{\mu}$ are the damaged material elastic moduli [24].

For a circular damage zone, Hutchinson computed the modification of the SIF for a mode I crack $[25]$ :

$$
\frac{K_{\text {damage }}}{K_{\text {undamage }}}=1-\frac{5}{8} \frac{1}{1-\nu}\left(\frac{\mu}{\bar{\mu}}-1\right)+\frac{3}{4} \frac{1}{1-\nu}\left(\bar{\nu} \frac{\mu}{\bar{\mu}}-\nu\right)
$$

This approximation is used to take into account the shielding effect due to the nucleation and growth of the microcracks.

\subsection{Parameters of the model}

The influence of the following parameters on the morphology of the simulated cracks is studied:

$-\rho=\frac{N}{x_{l} y_{l}} \frac{\pi s_{Q}^{2}}{4}$, the surface fraction of precipitates;

$-s_{0}$, the initial length of the microcrack;

while:

$-\sigma^{\text {ext }}$, the external stress.

$-v_{0}=0.1$, the microscopic dimensionless velocity of the macrocrack and of the microcracks;

- $K_{I}^{\text {th }}$, the propagation threshold into the matrix;

- $R_{\text {plast } 0}=0.1$, the distance below which the microcrack joins instantaneously with the macrocrack;

- $\sigma_{C}(i)=400 x_{i}+100$, the critical stress above which microcrack $i$ is created. $x_{i}$ is a random variable which belongs to $[0,1]$;

- the size of the sample $x_{l}=100$ and $y_{l}=10$.

are kept constant. 
For each condition, severals cracks are produced which correspond to various realisations of the same microstructure.

\subsection{Crack morphology parameters}

Both the roughness exponent and the spikiness of the simulated cracks are analysed in order to have their morphology characterized. The roughness exponent $\zeta$ is determined with "the variable band: width" method [26]. The following quantity is computed:

$$
\begin{aligned}
Z \max (r)= & <\max \left(z\left(r^{\prime}\right)\right)_{r_{o}<r^{\prime}<r_{o}+r}-\min \left(z\left(r^{\prime}\right)\right)_{r_{o}<r^{\prime}<r_{o}+r}>_{r_{o}} \\
& \text { with } Z_{\max }(r) \propto\left\{\begin{array}{cc}
r^{\zeta} & (r \ll \xi) \\
h_{\max } & (r \gg \xi)
\end{array}\right.
\end{aligned}
$$

where $r$ is the width of the window. $Z \max (r)$ is the difference between the maximun and the minimun heights $z$ within this window, averaged over all possible origins $r_{o}$ of the window belonging to the profile.

On the other hand, the spikiness $h_{\max } / \xi$ defined as the ratio of the saturation height $h_{\max }$ to the self-affine correlation length $\xi$ characterizes the steepness of the local slopes. It has been argued by Bouchaud et al. [12] that this ratio is related to the fracture toughness

$$
K_{I C} \simeq 2 \sqrt{\gamma E}\left(\frac{h_{\max }}{\xi}\right)^{\frac{1}{2}}
$$

This result is in qualitative agreement with the common observation that "shallow" fracture surfaces are associated to more brittle materials (rocks, glass, ...) while spikier ones are observed in the case of toughter materials ( metallic alloys for example). It will be used in the following where a correlation between this morphological parameter and the microstructure is performed.

\section{RESULTS}

The simulations are performed on a personnal computer PENTIUM 90 Mhz. So, in order to avoid prohibitive computation times, a small box size is chosen. The cracks produced with the principles explained in the previous section will be analysed here.

\subsection{Effect of the microstructural parameters}

This section is concerned with the influence of microstructural parameters such as the precipitates sizes or the density of microcrack nucleation sites on the crack morphology parameters.

To study the effect of the density, the simulations are performed for $N=1000$ to 3000 , corresponding to seven values of the surface fraction of precipitates, $\rho$, ranging from $19.6 \%$ to $58.9 \%$, and for $\frac{K_{l}}{K_{I}^{t h}}=2.42$. As can be seen in Fig 2 , the craks are self-affine and the roughness exponent $\zeta$ seems independant of $\rho$. Small variations of the measured exponent are due to the small size of the scaling domain. On the contrary, the spikiness evolves with $\rho$ in a non monotonic way and a maximum is observed for $\rho \simeq 49 \%$ which corresponds to $N=2500$. To study the effect of $s_{\circ}$, the simulations are performed for $N=1000$ and $\frac{K_{7}}{K_{I}^{t h}}=6.17$. As can be seen in Fig 4, crack deflection increases with the size of the inclusions: this is reflected in the variation of the spikiness from 0.033 for $s_{0}=0.05$ to 0.038 for $s_{o}=0.5$. 


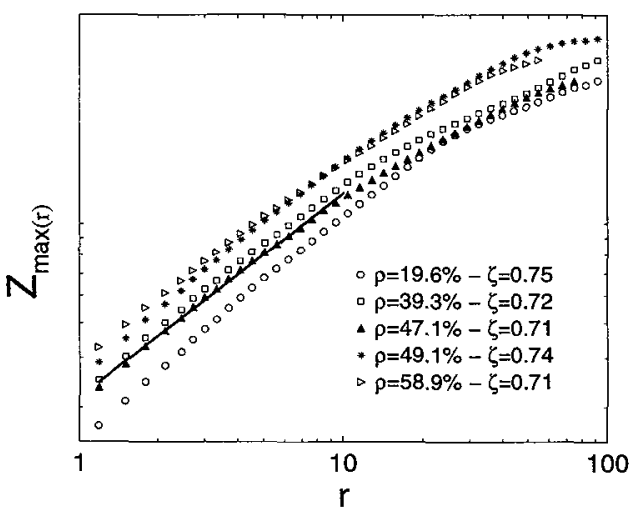

Figure 2: Evolution of $Z_{\max }(r)$ for various Figure 4: Evolution of the macrocrak deflecvalues of $N$

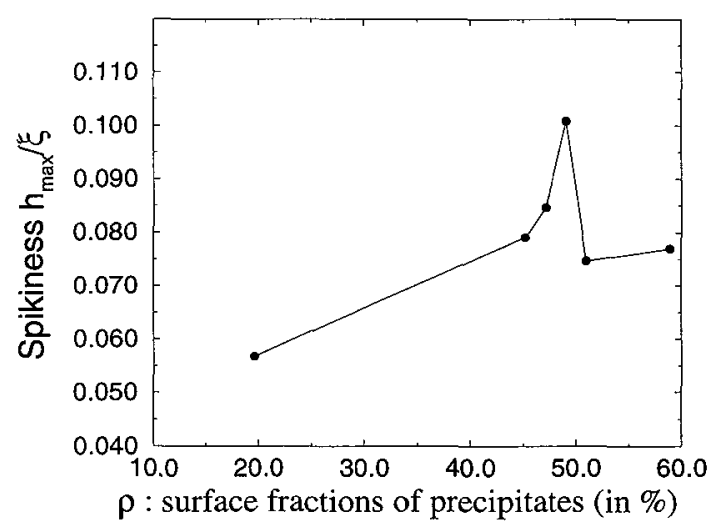

Figure 3: Evolution of the "spikiness" with the site density

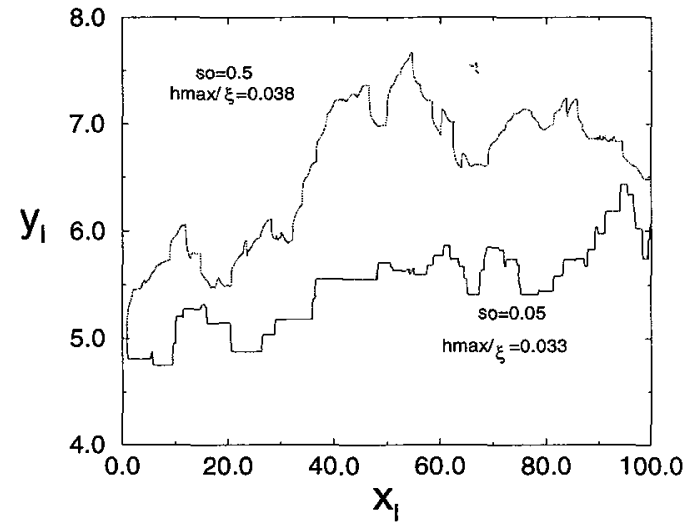

tion with the inclusions size

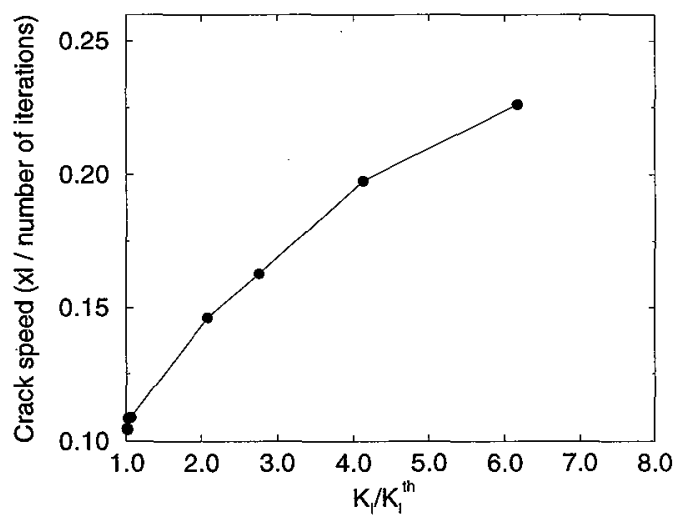

Figure 5: Evolution of the macrocrak speed with the applied stress 


\subsection{Effect of the external applied stress}

The influence of the applied stress on the roughness exponent, crack deflection and crack speed are analysed in this section. The results are obtained for $N=1000$ and $s_{0}=0.5$. As can be seen in Fig. 6, the effect of the external stress is not monotonic; as the stress is increased, crack deflection increases until the shielding effect becomes important and reduces this deflection.

Although the microscopic crack velocity $v_{0}$ is kept constant, the macroscopic macrocrack speed, defined as the ratio of the sample length to the number of iterations, increases with an increasing applied stress (see Fig 5).

Furthermore, the "effective" roughness exponent $\zeta$ (see Fig.6) increases with the external applied load, and hence with the "macroscopic" velocity of the main crack. This evolution could be interpreted as the existence of two fracture regimes with exponents close to 0.5 and 0.8 (or 0.7 ), separated by a crossover length $\xi_{C}$ decreasing with increasing crack velocity.

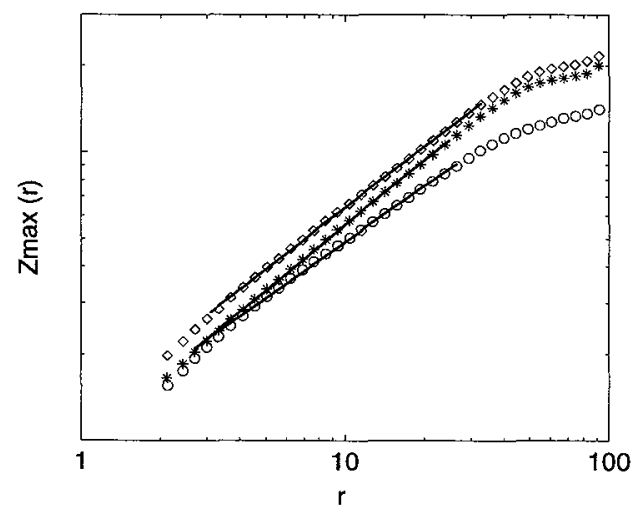

Figure 6: Evolution of the macrocrak deflection and the apparent roughness exponent with the applied stress

$\bullet: \frac{K_{r}}{K_{I}^{t_{h}}}=1.06, \zeta=0.65 \quad \diamond: \frac{K_{r}}{K_{I}^{t h}}=2.76, \zeta=0.71 *: \frac{K_{I}}{K_{I}^{t_{h}}}=6.17, \zeta=0.75$

\section{DISCUSSION}

Bidimensional cracks propagating in mode I through a heterogeneous material are simulated with a local model and their morphology is analysed. The cracks are self-affine, with a non-trivial roughness exponent $\zeta$. This index is clearly different from 0.5 (which corresponds to a random walk) or 1 (which corresponds to a regular line). As can be seen in Fig. 2, the roughness exponent seems independant of the microstructural parameters: the small variations could be due to the size of the scaling domain but this point should be checked for a greater sample size. This is in agreement with the experimental results. Furthermore, the average measured value of $\zeta$ is close to 0.7 , which is usually reported for experiments in two dimensions $[27,28,29,30]$.

On the contrary, the relevant length scales $h_{\max }$ and $\xi$ and their ratio $h_{\max } / \xi$ vary with the microstructural parameters. The optimal fracture toughness should be obtained for a surface fraction of secondary phases of $50 \%$. This behavior is due to a competition between two mechanisms due to the presence of microcracks: one is crack deflection, and the other one is the shielding. 
A systematic variation of $\zeta$ is nevertheless observed with increasing external loading. The exponent computed might well be an apparent exponent, corresponding to a mixing of the "quasi-static" $(\zeta \simeq 0.5)$ regime with the "kinetic" $(\zeta \simeq 0.7-0.8)$ regime. $\xi_{c}$ would then decrease with increasing the applied loading, and hence the crack velocity. However, such a determination over one decade of length scales is impossible. Increasing the size of the sample in order to extend the fractal domain should allow for a more accurate determination of $\zeta$.

Although this model indicates the existence of two fracture regimes, these cannot be defined properiy. As the simulated cracks can grow either at the microscopic velocity $v_{0}$ or more rapidly by junction with microcracks, we could imagine that the "quasi-static" regime should be related to a "sporadic" advance due to junctions whereas the "kinetic" regime could be associated to a more regular propagation or perhaps to a steady state junction regime. This hypothesis could be checked by either varying the ratio of the microcracks velocity to the macrocrack microscopic velocity, or varying the value of $R_{P l a s t}$. It seems experimentally [31] that the "quasi-static" regime could be linked to a discontinuous' growth of the crack whereas the "kinetic" regime seems to be related to a more continuous growth regime. On metallic alloys, fractured in fatigue, the "quasi-static" regime seems to correspond to a discontinuous propagation mode where the crack is not able to progress at every cycle. This might also be the case for the stress corrosion subcritical fracture of glass: the "quasi-static" regime corresponds to an intermittent fracture mode [32]. This is also what is described by the theoretical models $[17,18,19]$ where the depinning transition could also correspond to intermittency [33].

It would be interesting to study the influence of $s_{o}$ on the macrocrack morphology. The evolution of the spikiness reflects the well-known result that fracture toughness increases with the inclusions size. As can be seen in Fig. 4, the spikiness seems to increase with $s_{0}$ but a systematic study for various values of $N$ and $\sigma^{\text {ext }}$ should be performed. The evolution with $s_{o}$ of the crossover length $\xi_{c}$ between the "quasi-static" and "kinetic" regimes is also worth studying. Both evolutions could explain the apparent dependance sometimes reported between the measured roughness index and the microstructure.

\section{CONCLUSION}

A continuous numerical model of pure mode I macrocrack propagation into a two phases bidimensional material is presented. The morphological parameters of self-affine cracks produced for several mechanical and microstructural conditions are analysed. An optimum of the fracture toughness with the surface fraction of secondary phases is predicted by this model.

Aknowledgements: We are indebted to J.M. Dorvaux for his computational advices. We are particularly grateful to J.-P. Bouchaud, D. François and F. Creuzet for interesting discussions.

\section{References}

[1] B.B Mandelbrot and J.W. Van Ness, SIAM Rev. 10, 422 (1968)

[2] B.B. Mandelbrot, Phys. Scr. 32, 257 (1985) 
[3] M.B. Isichenko, Review of Modern Physics 64, 961 (1992)

[4] E. Bouchaud, G. Lapasset and J. Palnès, Europhys. Lett. 13, 73 (1990)

[5] P. Daguier, S. Hénaux, E. Bouchaud and F. Creuzet, to be published to Phys. Rev. E, (June 1995)

[6] J. Schmittbuhl, S. Gentier and S. Roux, Geophys. Lett. 20, 639 (1993)

[7] K.J. Maloy, A. Hansen, E.L. Hinrichsen and S. Roux, Phys. Rev. Lett. 68, 213 (1992)

[8] E. Guilloteau, thesis (1995)

[9] A. Nakano, R. Kalia and P. Vashishta, Phys. Rev. Lett., 73, 2336 (1994)

[10] B.B. Mandelbrot, D.E. Passoja and A.J. Paullay, Nature (London) 308, 721 (1984)

[11] J.J. Mecholsky, D.E. Passoja and K.S. Feinberg-Ringel, J. Am. Ceram. Soc. 72, 60 (1989)

[12] J.-P. Bouchaud, E. Bouchaud, G. Lapasset and J. Planès, Phys. Rev. Lett. 71, 2240 (1993)

[13] V.Y. Milman, R. Blumenfeld and N.A. Stelmashenko, R.C. Ball, Phys. Rev. Lett. 71, 204 (1993)

[14] V.Y. Milman, N.A. Stelmashenko and R. Blumenfeld, Prog. Mater. Sci. 38, 425 (1994)

[15] P. McAnulty, L.V. Meisel and P.J. Cote, Phys. Rev. A 46, 3523 (1992)

[16] E. Bouchaud and S. Navéos, J. Phys. I France, 5, 547 (1994)

[17] D. Ertas and M. Kardar, Phys. Rev. Lett. 69, 929 (1992)

[18] D. Ertas and M. Kardar, Phys. Rev. E, 48, 1228 (1993)

[19] D. Ertas and M. Kardar, Phys. Rev. Lett. 73, 1703 (1994)

[20] A. Nakano, R.K. Kalia and P. Vashishta, Phys. Rev. Lett. 75, 3138 (1995)

[21] L.R.F Rose, Int. Journal of fracture 31, 233 (1986)

[22] B. Cotterell and J.R. Rice, Int. journal of fracture 16, 155 (1980)

[23] H. Kitagawa, R. Yuuki and T. Ohira, Eng. Fracture Mechanics 7, 515 (1975)

[24] S. Nemat-Nasser and M. Hori Micromechanics: overall properties of heterogeneous materials, North-Holland (1993)

[25] J.W. Hutchinson, Acta Metall. 6, 1605 (1987)

[26] J. Schmittbuhl, J.P. Vilotte and S. Roux, Phys. Rev. E 51, 31 (1995)

[27] L. de Arcangelis, S. Redner and H.J. Herrmann, J. Physique (Paris), Lett. 46, L585 (1985)

[28] A. Hansen, E.L. Hinrichsen and S. Roux, Phys. Rev. Lett., 66, 2476 (1991)

[29] J. Kertesz, V. Horvath and F. Weber, Fractals, 1, 67 (1993)

[30] T. Engoy, K.J. Maloy, A. Hansen and S. Roux, Phys. Rev. Lett., 73, 834 (1994)

[31] P. Daguier, E. Bouchaud, B. Nguyen and F. Creuzet, in preparation (1996)

[32] B. Nguyen and F. Creuzet, in preparation (1996)

[33] P.B. Thomas and M. Paczuski, submitted to Phys. Rev. Lett. (1996) 